\title{
Estimation of Sidelobe Level Variations of Phased Codes in Presence of Random Interference for Bistatic Wideband Noise Radar
}

\author{
Ana Vazquez Alejos, ${ }^{1}$ Muhammad Dawood, ${ }^{2}$ and Habeeb Ur-Rahman Mohammed ${ }^{3}$ \\ ${ }^{1}$ The Signal Theory and Communication Department, University of Vigo, 36310 Vigo, Spain \\ ${ }^{2}$ The Klipsch School of Electrical and Computer Engineering, New Mexico State University, Las Cruces, NM 88001, USA \\ ${ }^{3}$ Texas Instruments Inc., Dallas, TX 75243, USA
}

Correspondence should be addressed to Ana Vazquez Alejos; analejos@uvigo.es

Received 22 July 2014; Accepted 3 November 2014

Academic Editor: Yong Bae Park

Copyright (C) 2015 Ana Vazquez Alejos et al. This is an open access article distributed under the Creative Commons Attribution License, which permits unrestricted use, distribution, and reproduction in any medium, provided the original work is properly cited.

\begin{abstract}
We discuss the importance of using the sidelobe level of the cross-correlation function as a criterion to implement a noise radar based on the transmission of wideband binary waveforms. Theoretical expressions are introduced for the parameters Peak-Sidelobe, Secondary-Sidelobe, and Integrated-Sidelobe levels for both Golay and pseudorandom binary sequences in presence of additive white Gaussian noise, relating the sequence length $M$ to the spectral power $N_{0}$ of the interfering noise. These expressions offer a valuable method for adaptive radar waveform design in order to determine sequence requirements which allow facing the noise present in the frequency band of interest. We also show a comparison of the ambiguity functions for Golay and PRBS sequences to analyze their performance in terms of Doppler and range accuracy. We describe a practical implementation of a pseudonoise waveform-based bistatic radar with reduced sidelobe level due to the use of Golay codes in combination with single side band modulation and operation at UHF band. Experimental measurements were performed in actual scenarios for ranging test of single and double targets. Linear polarizations were combined with different length sequences to determine their influence on the sounder performance under field test conditions.
\end{abstract}

\section{Introduction}

Radar data, consisting of returns from several objects, might result easily corrupted [1-4] because of the presence of noise and interferences, scattered signal components, and frequency dispersion [5-7] due to the random nature of the propagation medium. As a result of those impairment propagations, the target detection process is further complicated due to the problem of weak signals, which carry information about subtle changes that are buried in the sidelobes of stronger reflections.

At present, widely used noise radar systems operate with either very short pulses or linear frequency modulated waveforms and are based on either mono- or bistatic configurations [8]. These systems suffer from having strong sidelobes, thereby masking weaker returns from subtle changes and making it difficult to detect those variations due to a target presence.
In order to overcome the sidelobe problem, various coding techniques have been proposed with different degrees of success in [1-4]. One such technique is based on the transmission of pseudonoise (PN) waveforms, such as pseudorandom binary sequences (PRBS), and the system using them is known as noise radar [9-14]. Although PRBS are considered a good option in terms of their autocorrelation function, these sequences are not optimal if sidelobe level is taken into account. This problem can be overcome if the transmitting process is composed of codes showing good autocorrelation properties, mainly estimated in terms of sidelobe level amplitude. This is the case of the complementary binary series of sequences known as Golay series [9-14].

Golay complementary codes are a pair of equal length sequences that have the property of canceling the sidelobes when the autocorrelation functions corresponding to each sequence are algebraically added. As a consequence of this 
addition, the correlation peak is double to the one corresponding to the PRBS case, thereby providing a significant enhancement in the output signal-to-noise ratio. Also, once the individual autocorrelation functions are added, they provide zero sidelobes. These improvements are important when dealing with large attenuation and/or with stronger sidelobes, as is generally the case of subsurface, through-wall or through-dispersive media detections, or in applications that require the use of large frequency bandwidth.

Due to a priori knowledge of the real-time crosscorrelation function (CCF) properties of the transmitted binary sequences, an adaptive-on-transmit (AT) system can be derived for wideband radar systems using the information given by the Peak-Sidelobe (PSL), Secondary-Sidelobe (SSL), and Integrated-Sidelobe (ISL) as a design criterion.

This means that we can know the theoretical value of PSL, SSL, and ISL parameters at any instant for the transmitted code; besides, the generating conditions of the transmitted codes could be changed to improve the sidelobe values under the detection of a certain noise level presence. By adapting the sidelobe properties of the transmitted waveform to minimum levels, the overall performance of the system will achieve enhanced performance features to face anomalous or extreme operation conditions [15-18].

In this paper, we introduce a formal analysis of the sidelobe level trend for the phase codes PRBS and also for the complementary phase codes named Golay series. The ISL, SSL, and PSL parameters from their CCFs have been parameterized as functions of code length and interfering white Gaussian random noise power.

Additionally to the sidelobe issue, the noise radar technique presents some implementation requirements that often turn these systems into technical- or cost-unaffordable sounders. At the transmitter end, high bit rates and large sequence lengths are needed to achieve large amplitude and spatial resolution, respectively. This feature implies an analogue-digital conversion stage of great bandwidth and large amplitude resolution at the receiver end if a digital implementation is selected to build the sounder. This SDR (software defined radio) architecture may not be always easy to achieve and it usually results in very costly hardware.

In this paper, we introduce a solution to the problem caused by a large bandwidth requirement based on the use of single side band transmission that offers a double benefit: firstly, only half bandwidth will be required at the analoguedigital conversion stage of the receiver end, which will simplify the hardware and reduce costs; secondly, the traveling waveform will be exposed to less noise (interferences) levels, an especially important feature if the frequency band is radio electrically polluted, as the UHF band case.

Section 2 presents software simulations to illustrate a comparison of the robustness of PRBS and Golay sequences against noise interferences. Theoretical expressions are derived for PSL, SSL, and ISL terms, for both Golay and PRBS cases. Section 3 briefly describes the hardware implementation of the prototype system, followed by the experimental results in Section 4. Experimental measurements were carried out to perform ranging tests for single and double target identification for both sequences, demonstrating the viability of a phase code based noise radar in the UHF band. The influences of the sequence length, as well as the linear polarization used, have been analyzed in order to determine the behavior of this radar sounder under field test conditions. Finally, conclusions are offered in Section 5.

\section{Noise Radar Techniques Based on Phase Coded Binary Sequences}

The noise modulated radar technique offers a large number of advantages mainly due to its robustness to interferences $[8,18$, 19], and the wideband version received importance especially in the last decades $[13,14,17-19]$. Nevertheless, until not long ago, it was very difficult to find practical implementations of these systems. One of the main problems with them is that they show detection ambiguity zones and the presence of sidelobes that can mask weak reflections [8]. Another key element of the noise radar technique is the waveform generation that is mostly based on the transmission of a wideband signal that usually consists of monopulsed transmissions.

Other implementations of the wideband random noise technique use waveforms based on pseudorandom binary sequences with maximum length, also named PRBS sequences with length $M$ given in bits or chirps. This technique [8] offers great advantages regarding high resolution of targets as well as its great immunity to detection in hostile surroundings, as well as in presence of natural or man-made caused interferences.

Nevertheless, the radar technique by transmission of PRBS sequences presents a limitation in the offered dynamic range which goes bound to length $M$ of the transmitted sequence $[12,13]$. Thus, detecting weaker echoes is difficult, which may be confused with noise in some cases due to the large attenuation undergone by the propagated signal. In addition, PRBS sequences present a serious problem of large power sidelobes presence [10], which worsens the problem of false echoes detection that is usual in radar applications.

The sidelobe amplitude level is directly proportional to length $M$ of the sequence, so that if the length is increased with the purpose of increasing the dynamic range, the sidelobe amplitude level will be increased in counterpart. However, by increasing the length of the transmitted sequence, the speed of target detection is decreased, thus limiting the response speed of the radar device.

In this paper, we considered a solution to the problem produced by increasing the length $M$ of the transmitted PRBS sequence by considering the use of Golay series [9-11] which lead to a twofold dynamic range with respect to the one corresponding to a PRBS sequence with the same length $M$. This allows the use of smaller length sequences to increase the target detection speed. This feature compensates the need of transmitting two sequences in the Golay case that would increase the time needed for measurement.

In Section 2.1, the capabilities of PRBS and Golay sequences are measured in terms of PSL, SSL, and ISL levels. The effect of noise is presented in Section 2.2. This comparison also shows better understanding of advantages 
provided by reduced sidelobe levels. The results indicate which parameters of these binary sequences used in noise radar can be easily adapted depending on the operation requirements according to the idea of an AT system.

2.1. Theoretical Expressions: PSL, SSL, and ISL. The theoretical expressions have been derived for PSL, SSL, and ISL parameters definition, sequence length $M$, and the noise $N_{0}$ statistics mean $\mu_{N}$ and variance $\sigma_{N}$., for unitary amplitude level of the pulses $\pm 1 \mathrm{~V}$. The theoretical definition of the sidelobe level parameters for a signal $s(t)$ is given by

$$
\begin{gathered}
\mathrm{PSL}=\max \left\{R_{S}(\tau)\right\}-\max \left\{R_{S}(\tau)\right\} \\
=R_{S}(\tau=0)-\max \left\{R_{S}(\tau \neq 0)\right\}, \\
\mathrm{SSL}=\max \left\{R_{S}(\tau \neq 0)\right\}, \\
\mathrm{ISL}=\int_{-\infty}^{\infty} R_{S}(\tau \neq 0) .
\end{gathered}
$$

For the Golay case, the pair of sequences composing the code $g(t)$ are denoted as $g_{a}(t)$ and $g_{b}(t)$, with $0 \leq t \leq M \cdot T_{c}$. As we explained above, each of them has been added with the same noise signal $n(t)$ and later correlated with the original sequence. These operations can be expressed according to (2)-(4) where $\otimes$ indicates correlation:

$$
\begin{aligned}
R_{g n}(\tau)= & {\left[\left(g_{a}(t)+n(t)\right) \otimes g_{a}(t-\tau)\right] } \\
& +\left[\left(g_{b}(t)+n(t)\right) \otimes g_{b}(t-\tau)\right] \\
= & g_{a}(t) \otimes g_{a}(t-\tau)+g_{b}(t) \otimes g_{b}(t-\tau) \\
& +n(t) \otimes g_{a}(t-\tau)+n(t) \otimes g_{b}(t-\tau), \\
R_{g n}(\tau)= & R_{g_{a}}(\tau)+R_{g_{b}}(\tau)+R_{g_{a} n}(\tau)+R_{g_{b} n}(\tau) \\
= & R_{g}(\tau)+R_{g_{a} n}(\tau)+R_{g_{b} n}(\tau), \\
R_{g n}(\tau)= & \begin{cases}2 M+R_{g_{a} n}(0)+R_{g_{b} n}(0), & \tau=0, \\
R_{g_{a} n}(\tau)+R_{g_{b} n}(\tau), & \tau \neq 0 .\end{cases}
\end{aligned}
$$

Taking into account (2)-(3), (1) can be written for the Golay case as follows:

$$
\begin{aligned}
& \mathrm{PSL}=\max \left\{R_{g n}(\tau)\right\}-\max \left\{R_{g_{n}}(\tau)\right\} \\
&=R_{g n}(\tau=0)-\max \left\{R_{g_{n}}(\tau \neq 0)\right\} \\
&=2 M-\max \left\{R_{g_{n}}(\tau \neq 0)\right\} \\
&=2 M-\max \left\{R_{g_{a} n}(\tau \neq 0)+R_{g_{b} n}(\tau \neq 0)\right\}, \\
& \mathrm{SSL}=\max \left\{R_{g_{n}}(\tau \neq 0)\right\} \\
&=\max \left\{R_{g_{a} n}(\tau \neq 0)+R_{g_{b} n}(\tau \neq 0)\right\}, \\
& \mathrm{ISL}=\int_{-\infty}^{\infty} R_{g^{n}}(\tau \neq 0) .
\end{aligned}
$$

The discrete CCFs $R_{g_{a} n}[k]$ and $R_{g_{b} n}[k]$ are given by the following expressions:

$$
\begin{aligned}
& R_{g_{a} n}[k]=\sum_{m=1}^{M} a_{m} \cdot n_{m+k}^{*}<\frac{1}{M} \sum_{m=1}^{M}\left|a_{m} \cdot n_{m+k}^{*}\right|^{2} \\
&<\frac{1}{M} \sum_{m=1}^{M}\left|a_{m}\right|^{2} \cdot\left|n_{m}\right|^{2}=\frac{1}{M} \cdot M \cdot\left(N_{0}-\mu_{N}\right), \\
& R_{g_{a} n}[k]<\frac{1}{M} \cdot M \cdot \sum_{m=1}^{M}\left|n_{m}\right|^{2} \approx \bar{n}-\operatorname{var}(n) .
\end{aligned}
$$

From (7) we infer that the cross-correlation between a sequence of the pair and random noise is not dependent on index $k$. Finally, we reach the following expressions (8) for PSL, SSL, and ISL involving $M$ and $N_{0}$ :

PSL $[\mathrm{dB}]$

$$
\begin{gathered}
=\left\{\begin{array}{cc}
10 \cdot \log _{10}(2 M), \quad N_{0} \leq 6-10 \cdot \log _{10}(M), \\
10 \cdot \log _{10}(2 M)-2 \cdot\left[6-10 \cdot \log _{10}(M)-N_{0}\right], \\
6-10 \cdot \log _{10}(M) \leq N_{0} \leq 47 \mathrm{dBW}, \\
0, \quad N_{0} \geq 47 \mathrm{dBW},
\end{array}\right. \\
\text { SSL }[\mathrm{dB}]=N_{0}-\mu_{N}, \\
\text { ISL }[\mathrm{dB}]=\frac{N_{0}}{2} .
\end{gathered}
$$

For the PRBS case, only one sequence composes the code and it is denoted as $p(t)$, with $0 \leq t \leq M \cdot T_{c}$. The same noise signal $n(t)$ is added and the resulting noisy signal is correlated with the original sequence. These operations can be expressed according to (9) where $\otimes$ indicates correlation:

$$
\begin{aligned}
& R_{p n}(\tau)= {[(p(t)+n(t)) \otimes p(t-\tau)] } \\
&= p(t) \otimes p(t-\tau)+n(t) \otimes p(t-\tau), \\
& R_{p n}(\tau)=R_{p}(\tau)+R_{n p}(\tau), \\
& R_{p n}(\tau)= \begin{cases}M+R_{n p}(0), & \tau=0, \\
R_{n p}(\tau), & \tau \neq 0 .\end{cases}
\end{aligned}
$$

Taking into account (8), (1) can be written for the PRBS case as follows:

$$
\begin{aligned}
& \mathrm{PSL}=\max \left\{R_{p}(\tau)\right\}-\max \left\{R_{n p}(\tau)\right\} \\
&= R_{p}(\tau=0)-\max \left\{R_{n p}(\tau \neq 0)\right\} \\
&= M-\max \left\{R_{n p}(\tau \neq 0)\right\}, \\
& \mathrm{SSL}=\max \left\{R_{n p}(\tau \neq 0)\right\}, \\
& \mathrm{ISL}=\int_{-\infty}^{\infty} R_{n p}(\tau \neq 0) .
\end{aligned}
$$


Finally we reach the following expressions:

$$
\begin{aligned}
& \text { PSL }[\mathrm{dB}] \\
& = \begin{cases}10 \cdot \log _{10}(M), & N_{0} \leq 6-10 \cdot \log _{10}(M), \\
10 \cdot \log _{10}(2 M)-2 \cdot\left[6-10 \cdot \log _{10}(M)-N_{0}\right], \\
0, & 6-10 \cdot \log _{10}(M) \leq N_{0} \leq 47 \mathrm{dBW}, \\
0 & N_{0} \geq 47 \mathrm{dBW},\end{cases}
\end{aligned}
$$

SSL $[\mathrm{dB}]$

$$
=\left\{\begin{array}{c}
10 \cdot \log _{10}\left(\sqrt{\frac{3}{2}} \cdot M\right), \\
N_{0} \leq 6-10 \cdot \log _{10}(M), \\
-10 \cdot \log _{10}(M)+\left[6-10 \cdot \log _{10}(M)-N_{0}\right], \\
\quad 6-10 \cdot \log _{10}(M) \leq N_{0} \leq 47 \mathrm{dBW}, \\
0, \quad N_{0} \geq 47 \mathrm{dBW},
\end{array}\right.
$$

$$
\operatorname{ISL}[\mathrm{dB}]=\frac{N_{0}}{2}-10 \cdot \log _{10}(2 M) \text {. }
$$

The first observation to be inferred, when comparing PSL/SSL/ISL expressions for both types of codes, is that the PSL for the Golay case is not influenced by the AWGN as much as for the PRBS case. Moreover, the SSL in the Golay case depends only on the noise parameters, whereas it also depends on the inherent autocorrelation noise in the PRBS case. So, we conclude that an irreducible noise is present in the ACF for a PRBS sequence, the so-called code noise [18]. The same trend is observed for the ISL parameter.

2.2. Robustness against Sidelobe Presence. Software simulations using MATLAB have been performed to illustrate the robustness of PRBS and Golay sequences against noise interferences. For this purpose, two 4096-bit-length Golay sequences and one 8192-length PRBS sequence with amplitude level of $\pm 1 \mathrm{~V}$ and chip period $T_{c}=1 \mathrm{~s}$ were generated using software [14-16]. Additive white Gaussian noise (AWGN) was added to each sequence with power level $N_{0}$ within the range $[-100,+100] \mathrm{dBW}$. The added noise has the same bit rate as sequences used, thus offering identical bandwidth conditions.

Cross-correlation functions between noisy and original sequences were obtained. Later, PSL, SSL, and ISL levels were measured without performing any average that would aim to reduce the added noise. From the plots shown in Figure 1 the following conclusions can be inferred.

(i) For $N_{0}$ larger than $3 \mathrm{~dB}$, PSL levels in Golay and PRBS are the same.

(ii) SSL level in Golay sequences is almost $50 \mathrm{~dB}$ down compared to that in PRBS.

(iii) As the $N_{0}$ ratio increases, the SSL level difference between Golay and PRBS sequence decreases.

(iv) ISL level in the Golay case is almost $50 \mathrm{~dB}$ lesser than the ISL level for PRBS.

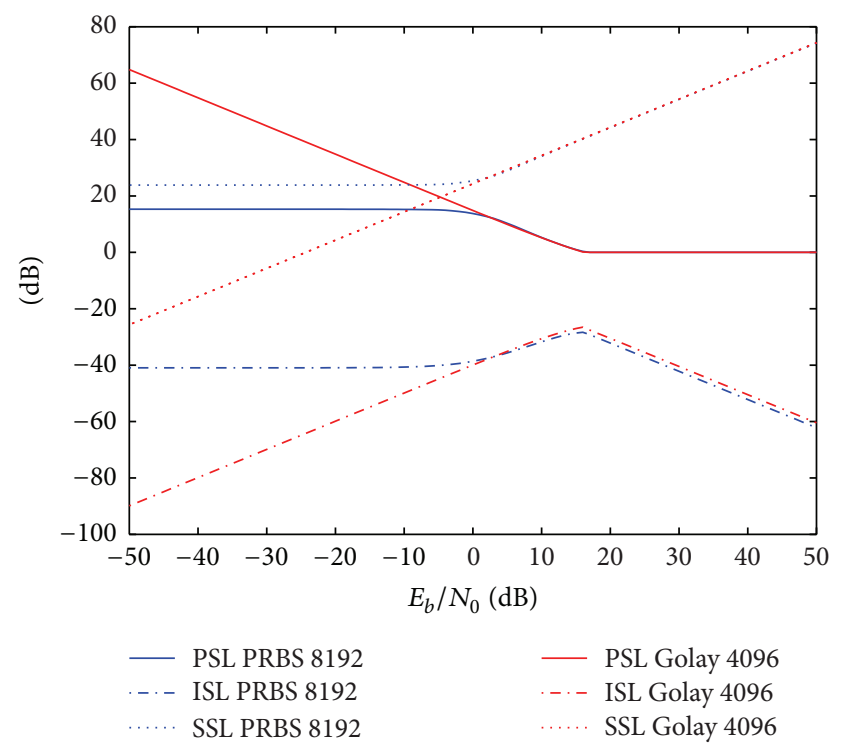

FIGURE 1: PSL, SSL, and ISL comparison for 4096-Golay and 8192PRBS sequences.

(v) As the $N_{0}$ ratio increases, the ISL level difference between the Golay and PRBS sequences decreases.

(vi) For $N_{0}$ equals $16 \mathrm{~dB}$, PSL level is zero. At this point the AWGN power is larger than the sequence power, so the noise masks the signal. This fact would correspond to a negative signal-to-noise ratio region.

If cross-correlation functions are averaged to obtain a noise effect reduction, the plots in Figure 2 are obtained. Among other differences, we can notice that when the case of zero noise is considered for the SSL curve, in the Golay case, the cross-correlation sidelobes are always cancelled regardless of the noise level and then the SSL value is constant and is only determined by the sequence length.

2.3. Ambiguity Functions Comparison. The ambiguity function for the complementary codes has been derived following the simplified method based on combining multiple range cuts $[17,19-21]$. According to this method, the formal expression for the ambiguity function of a complementary code waveform is given by

$$
\begin{aligned}
& \left|\chi\left(\tau ; f_{d}\right)\right| \\
& =\left|\int_{-\infty}^{\infty} s(t) \cdot s^{*}(t-\tau) \cdot e^{j 2 \pi f_{d} t} d t\right| \\
& =\mid \int_{-\infty}^{\infty} s_{a}(t) \cdot s_{a}^{*}(t-\tau) \cdot e^{j 2 \pi f_{d} t} d t \\
& \quad+\int_{-\infty}^{\infty} s_{b}(t) \cdot s_{b}^{*}(t-\tau) \cdot e^{j 2 \pi f_{d} t} d t \mid .
\end{aligned}
$$




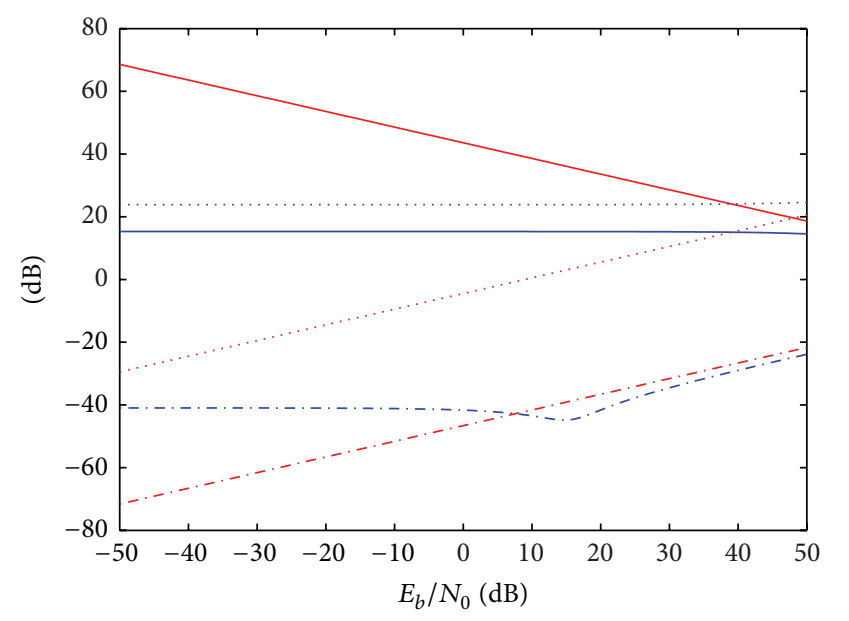

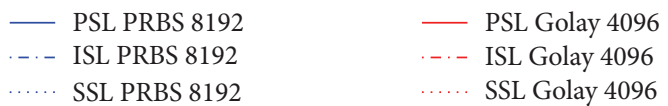

FIGURE 2: PSL, SSL, and ISL comparison for 4096-Golay and 8192PRBS sequences with averaged CCFs.

The above expression turns into (13) if Parseval's theorem is applied:

$$
\begin{aligned}
& \left|\chi\left(\tau ; f_{d}\right)\right| \\
& \quad=\left|\int_{-\infty}^{\infty} S^{*}(f) \cdot S\left(f-f_{d}\right) \cdot e^{-j 2 \pi f \tau} d f\right| \\
& =\mid \int_{-\infty}^{\infty} S_{a}^{*}(f) \cdot S_{a}\left(f-f_{d}\right) \cdot e^{j 2 \pi f \tau} d f \\
& \quad+\int_{-\infty}^{\infty} S_{b}^{*}(f) \cdot S_{b}\left(f-f_{d}\right) \cdot e^{-j 2 \pi f \tau} d f \mid .
\end{aligned}
$$

Equation (13) can be implemented in MATLAB developing the ambiguity function as a sequence of range cuts. Moreover, a simpler expression to be implemented can be derived from an arrangement of (12), which can be seen as a correlation of two functions:

$$
\begin{aligned}
\left|\chi\left(\tau ; f_{d}\right)\right| & =\left|\int_{-\infty}^{\infty} s(t) \cdot s^{*}(t-\tau) \cdot e^{j 2 \pi f_{d} \tau} d t\right| \\
& =\left|\left[s(\tau) \cdot e^{j 2 \pi f_{d} \tau}\right] \otimes s(\tau)\right| .
\end{aligned}
$$

The autocorrelation of complementary codes is achieved by adding two individual autocorrelation functions, so the term $s(\tau) \cdot e^{j 2 \pi f_{d} \tau} \otimes s(\tau)$ can be expressed as a composition of the correlation corresponding to each sequence of the pair:

$$
\begin{aligned}
\chi\left(\tau ; f_{d}\right)= & {\left[s_{a}(\tau) \cdot e^{j 2 \pi f_{d} \tau}\right] \otimes s_{a}(\tau) } \\
& +\left[s_{b}(\tau) \cdot e^{j 2 \pi f_{d} \tau}\right] \otimes s_{b}(\tau) .
\end{aligned}
$$

In the Fourier domain, (15) turns into (16) if we apply Parseval's theorem as in (13):

$$
\begin{aligned}
F\left\{\chi\left(\tau ; f_{d}\right)\right\}= & F\left\{\left[s_{a}(\tau) \cdot e^{j 2 \pi f_{d} \tau}\right] \otimes s_{a}(\tau)\right\} \\
& +F\left\{\left[s_{b}(\tau) \cdot e^{j 2 \pi f_{d} \tau}\right] \otimes s_{b}(\tau)\right\} .
\end{aligned}
$$

If we evaluate both Fourier transforms in (16) for a discrete interval of frequency values, $f_{d}=f_{k}$, and then we apply an IFFT and a modulus operator, we obtain the ambiguity function corresponding to the complementary codes by the method of range cuts. A comparison has been performed for the ambiguity functions corresponding to PRBS and Golay codes. A sequence length of $M=31$ was chosen for the PRBS case and $M=32$ for the Golay case, with pulse amplitude of $\pm 1 \mathrm{~V}$ and an oversampling factor equal to 100 . The outcomes are plotted in Figures 3 and 4, respectively.

Generally, we can observe a better performance in range estimation and Doppler tolerance for the Golay code in the contour plots. In the $\left|\chi\left(\tau ; f_{d}=0\right)\right|$ cuts we observe a null presence of sidelobes for the Golay case that agrees with the autocorrelation properties of these codes. In the PRBS sequence, larger sidelobe values are present.

In the following section, we describe a practical implementation of the Golay-based noise radar. Additionally, some details of the measurement procedure and signal processing are analyzed.

\section{Measurement System}

The proposed wideband noise radar consists of the transmission of a binary sequence, either PRBS or Golay. The sequence is digitally generated with the desired length and binary rate and modulated for transmission. A single side band transmission has been applied in order to reduce the bandwidth requirement in the analogue-digital conversion stage, and it is accomplished by using the transmitting antenna as a filter. In the reception and later processing, the phase component has been also considered and not only the envelope of the received signal [22]. For this purpose, a superheterodyne detection is carried out by means of a zero baseband downconversion combined with a $I / Q$ demodulation. A complete block diagram of the system can be seen in Figure 5 .

A software tool was developed in Labview to configure and control the PN generator and the oscilloscope, hence ensuring the correct data acquisition and recording. This tool ensures the impulse response snapshot to be measured in a short enough time in order for the channel response to remain essentially constant during acquisition.

The resultant measurement system is a wideband radio channel sounder in the time domain that uses the cross-correlation technique to obtain the complex impulsive response estimate or $h(t, \tau)$ [23]. From this function the range or distance to a target can be extracted from the delay information reported by the echoes detected in the CCF.

3.1. Parameters of the Implemented Sounder. The pulse pattern generator produced the binary Golay and PRBS sequences of $2 V_{\mathrm{pp}}$ amplitude with a maximum baseband 

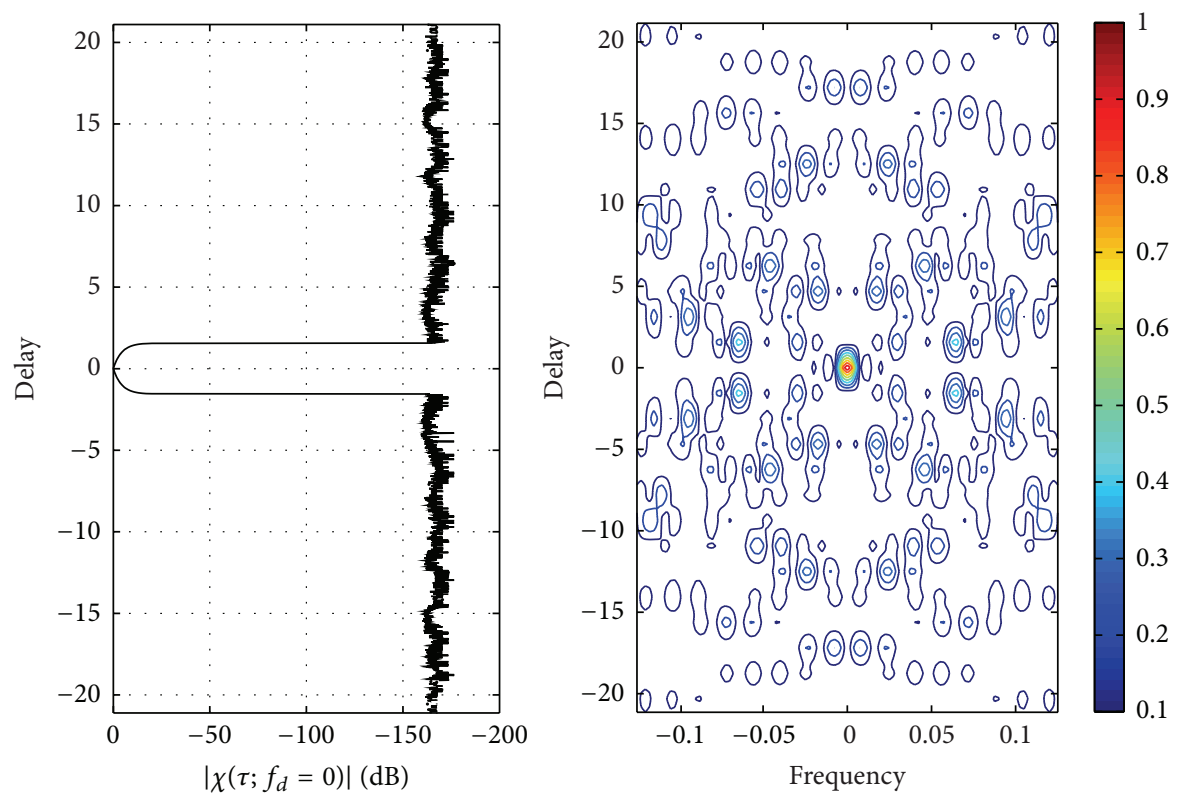

FIGURE 3: Ambiguity function for a 32-bit-length Golay code: contour plot and $\left|\chi\left(\tau ; f_{d}=0\right)\right|$ cut.
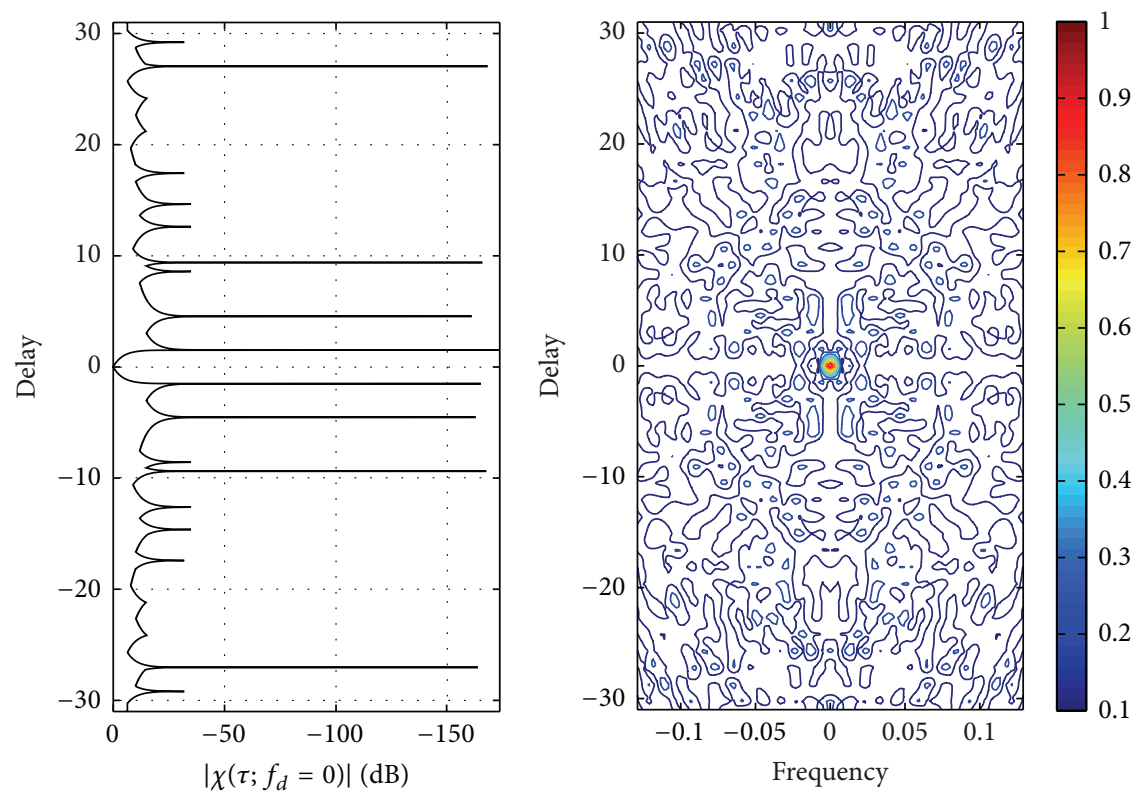

FIGURE 4: Ambiguity function for a 31-bit-length PRBS code: contour plot and $\left|\chi\left(\tau ; f_{d}=0\right)\right|$ cut.

frequency $f_{c}$ of $250 \mathrm{MHz}$ resulting in a chip period of $4 \mathrm{~ns}$ $\left(1 / f_{c}\right)$. As indicated in Figure 5 , this binary code was mixed with a frequency carrier $f_{0}$ of $500 \mathrm{MHz}$, which was also used in the receiver end to demodulate the incoming signal, so the output radio frequency (RF) band extended from $250 \mathrm{MHz}$ to $750 \mathrm{MHz}$. The RF modulated signal boosted an amplifier to obtain an output power of $+17 \mathrm{dBm}$ and was transmitted via a log periodic antenna operating from $500 \mathrm{MHz}$. Thus, only the upper band of the modulated signal, that is, $500 \mathrm{MHz}$ to $750 \mathrm{MHz}$, was transmitted. The radiating elements consisted of one quad ridged horn antenna, model WJ-8326-12, and a log antenna model AR7-19 also from Watking Johnson, arranging the first one in the receiver and the second one in the transmitter end. All the frequency generators, as well as the sequence generator and the digital oscilloscope, were phase synchronized by using a $10 \mathrm{MHz}$ rubidium oscillator as a reference clock.

Once conditioned, the received RF signal was fed to an $I / Q$ mixer to yield in-phase baseband (I) and quadraturephase $(Q)$ components of the baseband downconverted signal. The analog to digital conversion of the $250 \mathrm{MHz}$ baseband $I$ and $Q$ signals was made by an oscilloscope at a sampling rate of $1 \mathrm{GSample/s}$. That signal could later be resampled at higher or lower rates if needed. The sounder design has a 


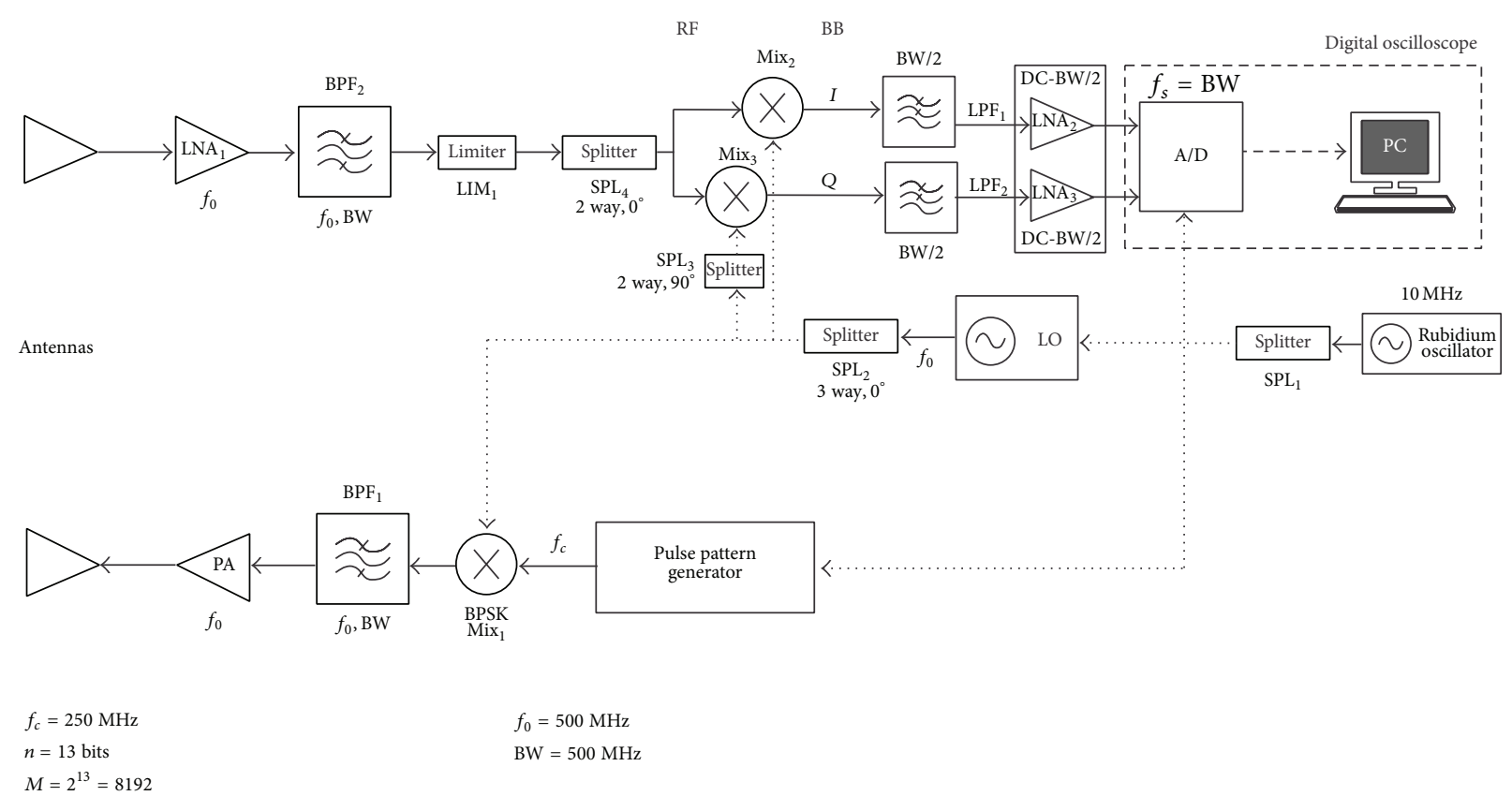

FIGURE 5: System block diagram.

TABLE 1: System parameters.

\begin{tabular}{lccc}
\hline Parameter & \multicolumn{3}{c}{ Value } \\
\hline $\begin{array}{l}\text { Register length }(m) \text { in bits } \\
\text { Code length } M\end{array}$ & 13 & 11 & 10 \\
$\quad$ PRBS: $M=2^{m}-1$ & 8191 & 4095 & 2047 \\
$\quad$ Golay: $M=2^{m}$ & 8192 & 4096 & 2048 \\
Code rate or chip period, $T c$ & $4 \mathrm{~ns}$ & $4 \mathrm{~ns}$ & $4 \mathrm{~ns}$ \\
Dynamic range & & & \\
$\quad$ PRBS & $39.13 \mathrm{~dB}$ & $36.13 \mathrm{~dB}$ & $33.13 \mathrm{~dB}$ \\
$\quad$ Golay & $42.14 \mathrm{~dB}$ & $39.13 \mathrm{~dB}$ & $36.13 \mathrm{~dB}$ \\
Delay resolution, $\Delta \tau$ & $8 \mathrm{~ns}$ & $8 \mathrm{~ns}$ & $8 \mathrm{~ns}$ \\
Maximum delay, $\tau_{\max }$ & & & \\
$\quad$ PRBS & $32.764 \mu \mathrm{s}$ & $16.380 \mu \mathrm{s}$ & $8.188 \mu \mathrm{s}$ \\
$\quad$ Golay & $32.768 \mu \mathrm{s}$ & $16.384 \mu \mathrm{s}$ & $8.192 \mu \mathrm{s}$ \\
Range resolution, $\Delta s$ & $1.2 \mathrm{~m}$ & $1.2 \mathrm{~m}$ & $1.2 \mathrm{~m}$ \\
Maximum spatial, $s_{\max }$ & & & \\
$\quad$ PRBS & $9829.2 \mathrm{~m}$ & $4914 \mathrm{~m}$ & $2456.4 \mathrm{~m}$ \\
$\quad$ Golay & $9830.4 \mathrm{~m}$ & $4915.2 \mathrm{~m}$ & $2457.6 \mathrm{~m}$ \\
\hline
\end{tabular}

noise figure of $3.11 \mathrm{~dB}$ and dynamic range of $80.84 \mathrm{~dB}$ at room temperature $290 \mathrm{~K}$. In Table 1 we have summarized the main features of the salient system. The parameters that indicate the detection capability of the implemented sounder are listed in Table $2[14,23]$.

\section{Experimental Measurements}

Field tests were performed for determining the range or distance to a target; firstly, they were conducted under controlled conditions inside an anechoic chamber, and, later, the
TABle 2: Design system specifications.

\begin{tabular}{lc}
\hline Parameter & Value \\
\hline Carrier Frequency & $500 \mathrm{MHz}$ \\
Baseband & $\mathrm{DC}-250 \mathrm{MHz}$ \\
Frequency range & $500-750 \mathrm{MHz}$ \\
Noise figure & $3.11 \mathrm{~dB}$ \\
Receiver output noise & $-83.9 \mathrm{~dB}$ \\
Dynamic range & $80.84 \mathrm{~dB}$ \\
Sampling frequency & $1 \mathrm{GSa} / \mathrm{s}$ \\
Transmitting antenna gain & $7.31 \mathrm{dBi}$ \\
Receiving antenna gain & $5 \mathrm{dBi}$ \\
\hline
\end{tabular}

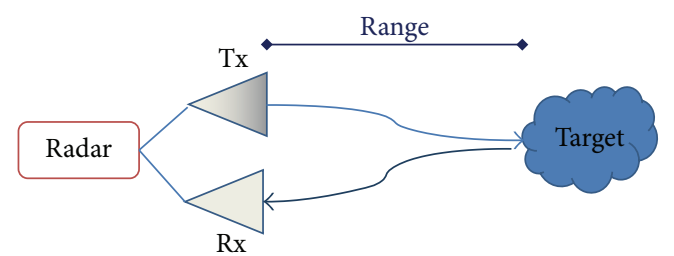

FiguRE 6: Bistatic radar configuration for measurement setup.

experiment was repeated in one outdoor scenario (top-roof) with different link ranges, for single and double target detection. Orthogonal linear polarizations were used to analyze a possible influence of polarization on results. Transmitter, receiver, and target locations were chosen to provide line of sight (LOS). A general schematic of the measurement setup used for the outdoor tests is given in Figure 6. It can be seen that it corresponds to a classical bistatic radar configuration. 


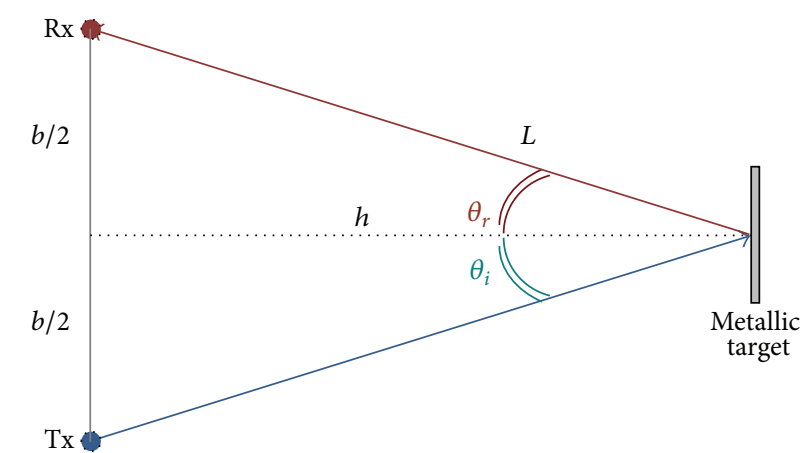

FIGURE 7: Geometry setup for single target detection measurement. For ranges 1 and $2, b=2.25 \mathrm{~m}, h=14.22 \mathrm{~m}, L=14.4 \mathrm{~m}$, and $\theta_{i}=$ $4.45^{\circ}$; for range $3, b=3.35 \mathrm{~m}, h=31.07 \mathrm{~m}, L=37.6 \mathrm{~m}$, and $\theta_{i}=$ $2.55^{\circ}$.

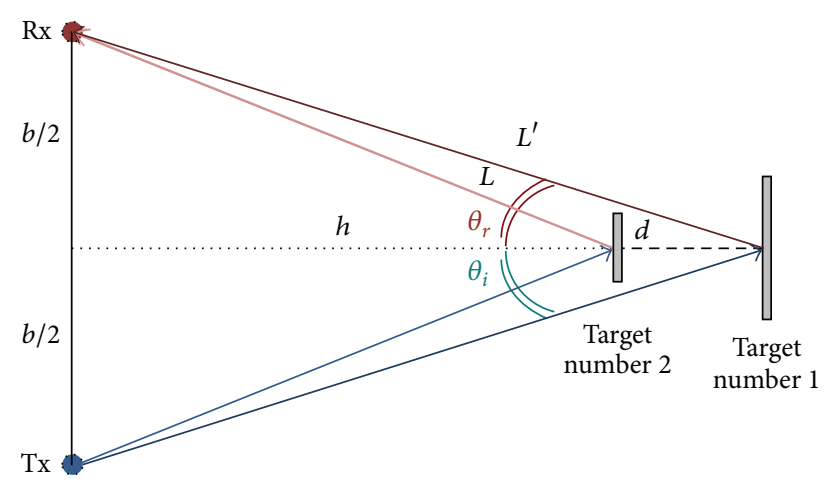

FIGURE 8: Geometry setup for double target detection measurement: $b=3.35 \mathrm{~m}, h=31.07 \mathrm{~m}, L=31.1 \mathrm{~m}, L^{\prime}=37.6 \mathrm{~m}, \theta_{i}=2.55^{\circ}$, and $d=6.5 \mathrm{~m}$.

A single target detection experiment was conducted firstly. The geometry for this measurement setup is shown in Figure 7. Secondly, a double target experiment was driven according to the geometry shown in Figure 8. This second target was also an aluminum plate with smaller dimensions, $0.315 \mathrm{~m}^{2}$.

PRBS and Golay sequences were generated, modulated, and later single side band transmitted in order to obtain a performance comparison in terms of range estimation and PSL, SSL, and ISL values achieved. The transmission is not in continuous mode, but it is armed with an external signal that indicates when transmission starts; once the sequence starts being transmitted by the programmable digital generator, its trigger output is activated; further, this signal triggers the acquisition in the oscilloscope.

The two-way measured distance between transmitting and receiving antenna can be obtained according to two different procedures. Firstly, by measuring the delay observed between the received signals with respect to the delay present in a back-to-back connection. The second one consists of performing a cross-correlation between the receiving signals and an ideal version of the transmitted waveform. The output of this cross-correlation is the impulsive response estimate that contains the detected multipath components and the

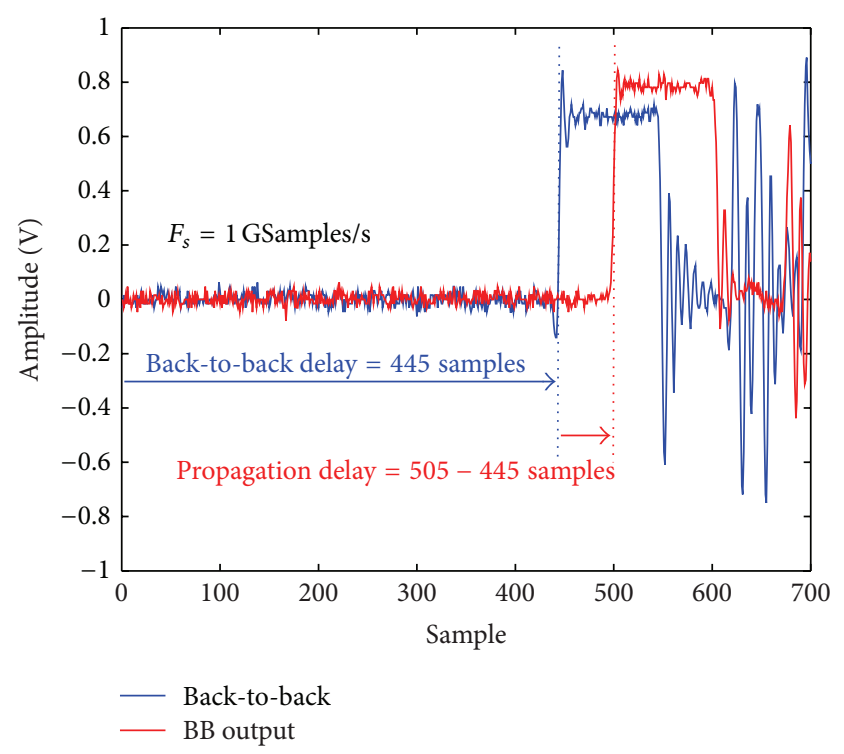

FIGURE 9: Propagation delay estimation for determining link range in the anechoic chamber.

excess delay value corresponding to each one of them. From this relative delay value, the two-way range can be derived adding the delay corresponding to the back-to-back connection.

Different sequence lengths have been used; for the Golay case it was always chosen a sequence with half the length than the one corresponding to PRBS code. This allows performing a comparison in the same conditions of dynamic range level, but it also compensates for the need of a double measurement time due to transmitting two sequences in the Golay case. The measurement procedure has been firstly used for an 8192length PRBS sequence followed by a 4096-length Golay code. Then it was repeated for a 4096-PRBS with a 2048-Golay code.

4.1. Anechoic Chamber. A simple range test was performed in order to ensure a proper functioning of the sounder. This experiment consisted of placing the transmitter and receiver antennas in opposite sides of the anechoic chamber facing each other and with a separation distance of $8.68 \mathrm{~m}$. The height of the transmitter and receiver antenna was $1.5 \mathrm{~m}$. Experiments were performed when both the antennas were copolarized, that is, horizontal $(\mathrm{HH})$ and vertical (VV) polarization.

The two-way measured distance between transmitting and receiving antenna was $17.36 \mathrm{~m}$. Figure 9 shows the received signal when the system is connected back-to-back and the received $I / Q$ signals when the anechoic chamber setup is utilized. The measured delay due to the anechoic chamber length was 60.445 samples, which gives a time delay of $60 \mathrm{nsec}$ for a sampling frequency of $1 \mathrm{GSa} / \mathrm{s}$. Thus a roundtrip delay of $18 \mathrm{~m}$ was calculated representing an error of $1.8 \%$ for both polarization cases. 
4.2. Single Target. We selected a wide rectangular terrace placed on a building roof within the NMSU Campus to ensure open field propagation and quasistatic conditions. Three combinations of transmitter, receiver, and target locations were considered. The transmitter-receiver set stayed in the same position on the terrace, $b$ meters apart, whereas the target was placed in two different locations. Later, the distance between the transmitter-receiver sets was increased from $2.25 \mathrm{~m}$ to $3.35 \mathrm{~m}$.

These three situations created three different range links. The two-way distance between the sounder and the target was about $28.8,42$, and 75.2 meters for the three ranges. The height of the transmitter and receiver antenna was the same for all the cases, $1.8 \mathrm{~m}$. The target was an aluminum plate with dimensions $1.26 \mathrm{~m}^{2}$.

4.3. Double Target. Taking as a base the third configuration used in the single target experiment corresponding to a twoway range of 75.2 meters, a second metallic laminate was placed in front of the previous target. This second target was separated by $6.5 \mathrm{~m}$ from the first one, as depicted in Figure 8, and their dimensions were smaller $0.25 \cdot 1.26=0.315 \mathrm{~m}^{2}$.

From the first echo delay we will infer the two-way distance travelled from the transmitter to the receiver, via reflection on the second target (smaller), whereas the second echo is related to the range of the first target (larger).

\section{Experimental Results}

The received signals were offline processed by estimating the cross-correlation of the recorded signal with an ideal version of the transmitted waveform. The results for both PRBS and Golay sequences were obtained and the comparison between them was done in terms of range accuracy estimation and detected sidelobe level presence. Tables 3 and 4 summarize results achieved for the PSL, SSL, and ISL level derived from field measurements. Large values of radio interferences have been detected along the field test due to the pollution present in the UHF band in which the experiments were conducted.

The values corresponding to the anechoic chamber were reduced to simple link range estimation. As we indicated in above Section 4.1, a round-trip link was calculated with an error of $1.8 \%$ for both polarization cases. The value of this test was to demonstrate the proper functioning of the radar sounder.

For the outdoor experimental tests it can be observed that with larger code lengths, the values for PSL, SSL, and ISL also tend to increase. Some coherence loss can be appreciated in these results for both polarizations that are due to the large level interferences found in the UHF band. These interferences degrade the correlation functions, as indicated in Figures 1 and 2 and as explained in Section 2.1. Furthermore, for the longest range link, ground reflection can be the main reason for the correlation distortion.

The experiments were driven in actual open field conditions, so the calculated sidelobe levels integrated in the PSL, SSL, and ISL parameters do not only correspond to the inherent sidelobe level of the code but also correspond to
TABLE 3: Results for PSL, SSL, and ISL parameters-PRBS case.

\begin{tabular}{lcccccc}
\hline $\begin{array}{l}\text { Sequence transmitted } \\
M \text { (sequence length) }\end{array}$ & \multicolumn{5}{c}{4096} & \multicolumn{3}{c}{8192} \\
\hline $\begin{array}{l}\text { Link range [m] } \\
\text { PSL [dB] }\end{array}$ & $28.8 \mathrm{~m}$ & $42 \mathrm{~m}$ & $75.2 \mathrm{~m}$ & $28.8 \mathrm{~m}$ & $42 \mathrm{~m}$ & $75.2 \mathrm{~m}$ \\
$\quad$ Vertical & 4.95 & 0.034 & 2.86 & 4.49 & 0.6 & 1.43 \\
$\quad$ Horizontal & 2.93 & 2.12 & 3.49 & 2.14 & 1.51 & 3.68 \\
$\quad$ SSL [dB] & & & & & & \\
$\quad$ Vertical & 38.83 & 40.93 & 38.58 & 41.91 & 43.92 & 42.70 \\
$\quad$ Horizontal & 38.83 & 39.87 & 35.73 & 42.00 & 43.08 & 37.10 \\
ISL [dB] & & & & & & \\
$\quad$ Vertical & 22.98 & 22.64 & 21.79 & 24.1 & 23.84 & 22.96 \\
$\quad$ Horizontal & 24.73 & 23.37 & 23.67 & 25.45 & 24.77 & 26.78 \\
\hline
\end{tabular}

TABLE 4: Results for PSL, SSL, and ISL parameters-Golay case.

\begin{tabular}{|c|c|c|c|c|c|c|}
\hline \multirow{2}{*}{$\begin{array}{l}\text { Sequence transmitted } \\
M \text { (sequence length) }\end{array}$} & \multicolumn{6}{|c|}{ Golay } \\
\hline & & 2048 & & & 4096 & \\
\hline Link range $[\mathrm{m}]$ & $28.8 \mathrm{~m}$ & $42 \mathrm{~m}$ & $75.2 \mathrm{~m}$ & $28.8 \mathrm{~m}$ & $42 \mathrm{~m}$ & $75.2 \mathrm{~m}$ \\
\hline \multicolumn{7}{|l|}{ PSL $[\mathrm{dB}]$} \\
\hline Vertical & 7.02 & 3.56 & 6.65 & 7.30 & 4.92 & 4.86 \\
\hline Horizontal & 5.04 & 3.39 & 6.73 & 4.52 & 3.25 & 6.70 \\
\hline \multicolumn{7}{|l|}{ SSL $[\mathrm{dB}]$} \\
\hline Vertical & 37.00 & 37.30 & 34.93 & 39.62 & 39.25 & 39.65 \\
\hline Horizontal & 37.52 & 38.02 & 32.56 & 40.96 & 41.47 & 35.28 \\
\hline \multicolumn{7}{|l|}{ ISL $[\mathrm{dB}]$} \\
\hline Vertical & 20.32 & 20.19 & 19.60 & 21.47 & 21.13 & 20.32 \\
\hline Horizontal & 20.95 & 21.29 & 22.55 & 22.71 & 21.81 & 24.26 \\
\hline
\end{tabular}

actual multipath components. By enlarging the range link, we changed the environment conditions so new elements could appear in the area illuminated by the radar, that is, a wider field of view. Only free-scatterer environments could ensure that all the integrated values really corresponded to sidelobes presence.

Despite these facts, a general trend is clearly detected. According to results shown in Tables 3 and 4, Golay offers an overall better performance in terms of sidelobe problem, reaching values up to $4.32 \mathrm{~dB}$ larger for PSL ( $M=4096,42 \mathrm{~m}$ range, vertical polarization), $4.67 \mathrm{~dB}$ less for the ISL parameter $(M=4096,42 \mathrm{~m}$ range, vertical polarization), and $3.78 \mathrm{~dB}$ less in the ISL ( $M=4096,42 \mathrm{~m}$ range, horizontal polarization).

It was determined that range estimation worked with more accuracy for the Golay case, whereas PRBS reaches a relative error of $2.1 \%$ for the $28.8 \mathrm{~m}$ range and the combination 8192-PRBS with 4096-Golay, in both polarization cases. This result agrees with the lower PSL level calculated from the corresponding acquired snapshots, which offered a difference of $2.74 \mathrm{~dB}$ for the same parameter in the Golay case.

Based on the outcomes, the polarization influence on sidelobe detection shows lower PSL and larger SSL/ISL levels for the vertical case for ranges 1 and 2 . In the range estimation, as per Tables 5 and 6 , the polarization has provided similar results in the single target case, but outcomes seem to be more 
TABLE 5: Range estimation results-single target case.

\begin{tabular}{lcccccc}
\hline Sequence transmitted & \multicolumn{3}{c}{ Golay } & \multicolumn{3}{c}{ PRBS } \\
$M$ (sequence length) & \multicolumn{3}{c}{4096} & & & 8192 \\
\hline Link range [m] & 28.8 & 42 & 75.2 & 28.8 & 42 & 75.2 \\
Link range [ns] & 96 & 140 & 250.67 & 96 & 140 & 250.67 \\
Measured delay [ns] & & & & & & \\
$\quad$ Vertical & 97 & 141 & 251.67 & 94 & 138 & 254.67 \\
$\quad$ Horizontal & 97 & 142 & 251.67 & 94 & 138 & 254.67 \\
Estimated range [m] & & & & & & \\
$\quad$ Vertical & 29.1 & 42.3 & 75.5 & 28.2 & 41.4 & 76.4 \\
$\quad$ Horizontal & 29.1 & 42.6 & 75.5 & 28.2 & 41.4 & 76.4 \\
Relative error [\%] & & & & & & \\
$\quad$ Vertical & 1.04 & 0.71 & 0.4 & 2.1 & 1.43 & 1.6 \\
$\quad$ Horizontal & 1.04 & 1.43 & 0.4 & 2.1 & 1.43 & 1.6 \\
\hline
\end{tabular}

TABLE 6: Range estimation results-double target case.

\begin{tabular}{lrrrrr}
\hline Code transmitted & \multicolumn{2}{c}{ PRBS } & \multicolumn{3}{c}{ Golay } \\
$M$ (sequence length) & 4096 & 8192 & 2048 & 4096 & 8192 \\
\hline $\begin{array}{l}\text { Measured delay (ns) } \\
\quad \text { Horizontal }\end{array}$ & 42 & 40 & 44 & 44 & 42 \\
$\quad$ Vertical & 42 & 42 & 44 & 44 & 44 \\
$\quad$ Relative error (\%) & & & & & \\
$\quad$ Horizontal & 2.96 & 7.58 & 1.66 & 1.66 & 2.96 \\
$\quad$ Vertical & 2.96 & 2.96 & 1.66 & 1.66 & 1.66 \\
\hline
\end{tabular}

accurate for the vertical polarization in the double target results; on the other hand, the horizontal polarization reaches a range estimation relative error of $7.54 \%$ for the combination 8192-PRBS with 4096-Golay.

\section{Conclusions}

A bistatic radar system operating in the UHF band according to the wideband noise principle was built for experimental ranging tests. Theoretical simulations were conducted to demonstrate the influence of noise on a wideband noise radar performance while using PRBS or Golay codes. Robustness against noise was theoretically determined in terms of PSL, SSL, and ISL values.

A simplification is presented for the ambiguity functions of complementary code based waveform. According to the expression reached for Golay, a comparison between Golay and PRBS sequences was obtained. The results indicate a better performance in Doppler and range accuracy for the complementary codes as shown in the contour plots of the ambiguity function. The outcomes also indicate a good agreement with the autocorrelation properties of the respective codes, especially in the $\left|\chi\left(\tau ; f_{d}=0\right)\right|$ cuts.

Field tests were driven on the roof top of Thomas and Brown building at NMSU. Different ranges have been successfully determined, not only for single but also for double target experiments. Results for both PRBS and Golay sequences were obtained and the comparison between them was done in terms of range estimation and values for PSL,
SSL, and ISL parameters. Our experimental tests indicated that the Golay codes seem to offer lower sidelobe level than PRBS sequences even when a ratio $2: 1$ is observed in the length selection of the involved sequences.

Generally speaking, we have shown improvement of noise radar system performance by using Golay-based sequences over PRBS in four terms: SSL and ISL levels reduction, better dynamic range, better range estimation, and minimized sidelobe masking problem observed on the PSL parameter value tendency. These results are important in the context of a largely radio polluted band in actual open field conditions. This opens the door to future work; a method that improves the wideband noise radar performance in noisy conditions should be developed.

An adaptive-on-transmit (AT) system can be derived for wideband radar systems using the information given by the PSL, SSL, and ISL as a design criterion. The minimal out-ofband sidelobe (OBS) level has been pointed out as an additional criterion to choose the correct transmitting waveform $[18,19]$.

\section{Conflict of Interests}

The authors declare that there is no conflict of interests regarding the publication of this paper.

\section{Acknowledgments}

The authors dedicate this paper to the memory of their respected professor Dr. Russell Paul Jedlicka, demised on March 11, 2008. The authors thank the funding and support of Klipsch School of Electrical and Computer Engineering, New Mexico State University, and the Xunta de Galicia (Grant EMR2012/238).

\section{References}

[1] B. L. Lewis and J. Kretschmer, "A new class of polyphase pulse compression codes and techniques," IEEE Transactions on Aerospace and Electronic Systems, vol. 17, no. 3, pp. 364-372, 1981.

[2] B. L. Lewis and F. F. Kretschmer Jr., "Linear frequency modulation derived polyphase pulse compression codes and techniques," IEEE Transactions on Aerospace and Electronics Systems, vol. 18 , no. $5,1981$.

[3] W. K. Lee, H. D. Griffiths, and L. Vinagre, "Developments in radar waveform design," in Proceedings of the 12th International Conference on Microwaves and Radar (MIKON '98), vol. 4, pp. 56-76, May 1998.

[4] W. K. Lee, H. D. Griffiths, and R. Benjamin, "Integrated sidelobe energy reduction technique using optimal polyphase codes," Electronics Letters, vol. 35, no. 24, pp. 2090-2091, 1999.

[5] A. V. Alejos and M. Dawood, "Estimation of power extinction factor in presence of brillouin precursor formation through dispersive media," Journal of Electromagnetic Waves and Applications, vol. 25, no. 4, pp. 455-465, 2011.

[6] A. V. Alejos, M. Dawood, and L. Medina, "Experimental dynamical evolution of the brillouin precursor for broadband wireless communication through vegetation," Progress in Electromagnetics Research, vol. 111, pp. 291-309, 2011. 
[7] A. V. Alejos and M. Dawood, "Information retrieval and crosscorrelation function analysis of random noise radar signal through dispersive media," in Radar Sensor Technology XVI, vol. 8361 of Proceedings of SPIE, Baltimore, Md, USA, April 2012.

[8] M. Dawood, N. Quraishi, and A. V. Alejos, "Super-resolution doppler estimation using UWB random noise signals and MUSIC," IEEE Transactions on Aerospace and Electronic Systems, vol. 49, no. 1, pp. 325-340, 2013.

[9] M. Golay, “Complementary series," IEEE Transactions on Information Theory, vol. 24, pp. 82-87, 1961.

[10] R. Sivaswamy, "Multiphase complementary codes," IEEE Transactions on Information Theory, vol. 24, no. 5, pp. 546-552, 1978.

[11] S. Budisin, "Golay complementary sequences are superior to PN sequences," in Proceedings of the IEEE International Conference on Systems Engineering, pp. 101-104, September 1992.

[12] D. Daniels, Ground Penetrating Radar, Institution of Engineering and Technology, 2nd edition, 2004.

[13] R. M. Narayanan, X. Xu, and J. A. Henning, "Radar penetration imaging using ultra-wideband (UWB) random noise waveforms," IEE Proceedings: Radar, Sonar and Navigation, vol. 151, no. 3, pp. 143-148, 2004.

[14] A. V. Alejos, M. G. Sanchez, and I. Cuinas, "Improvement of wideband radio channel swept time-delay cross-correlation sounders by using golay sequences," IEEE Transactions on Vehicular Technology, vol. 56, no. 1, pp. 362-368, 2007.

[15] C.-Y. Chen, C.-H. Wang, and C.-C. Chao, "Complete complementary codes and generalized Reed-Muller codes," IEEE Communications Letters, vol. 12, no. 11, pp. 849-851, 2008.

[16] F. Fiedler, J. Jedwab, and M. G. Parker, "A framework for the construction of Golay sequences," IEEE Transactions on Information Theory, vol. 54, no. 7, pp. 3114-3129, 2008.

[17] M. Dawood and R. M. Narayanan, "Generalised wideband ambiguity function of a coherent ultrawideband random noise radar," IEE Proceedings: Radar, Sonar and Navigation, vol. 150, no. 5, pp. 379-386, 2003.

[18] A. V. Alejos, M. Dawood, and M. G. Sanchez, "Extended optimal filters for adaptive-on-transmit radar systems using binary codes," Progress in Electromagnetics Research, vol. 130, pp. 41-46, 2012.

[19] N. Levanon and E. Mozeson, Radar Signals, John Wiley \& Sons, New York, NY, USA, 2000.

[20] R. Turyn, "Ambiguity functions of complementary series," IEEE Transactions on Information Theory, vol. II-8, pp. 46-47, 1963.

[21] B. R. Mahafza, Radar Systems Analysis and Design Using Matlab, Chapman and Hall CRC, Boca Raton, Fla, USA, 2005.

[22] A. V. Alejos, M. Dawood, M. G. Sanchez, I. C. Gomez, R. Jedlicka, and H. U. Mohammed, "Radar de penetración en tierra mediante transmisión de formas de onda basadas en series de secuencias de fase complementaria," Patent no. P200701181, 2012.

[23] P. A. Bello, "Characterization of randomly time-variant linear channels," IEEE Transactions on Communication Systems, vol. CS-11, pp. 360-393, 1963. 

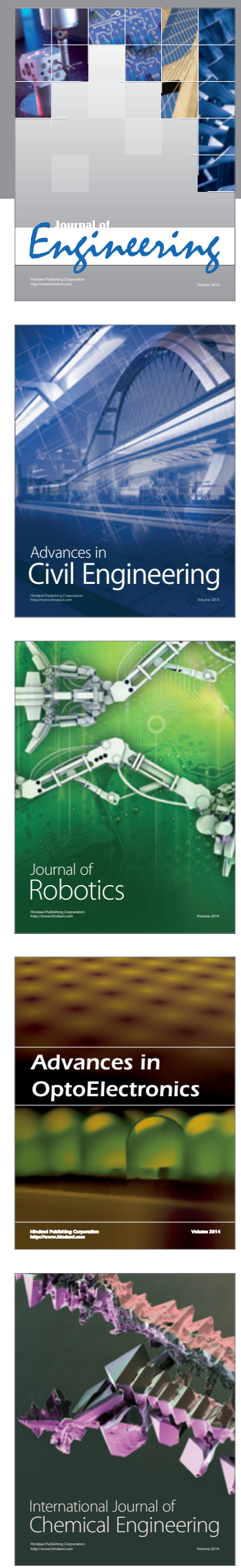

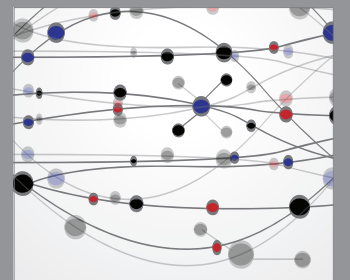

The Scientific World Journal
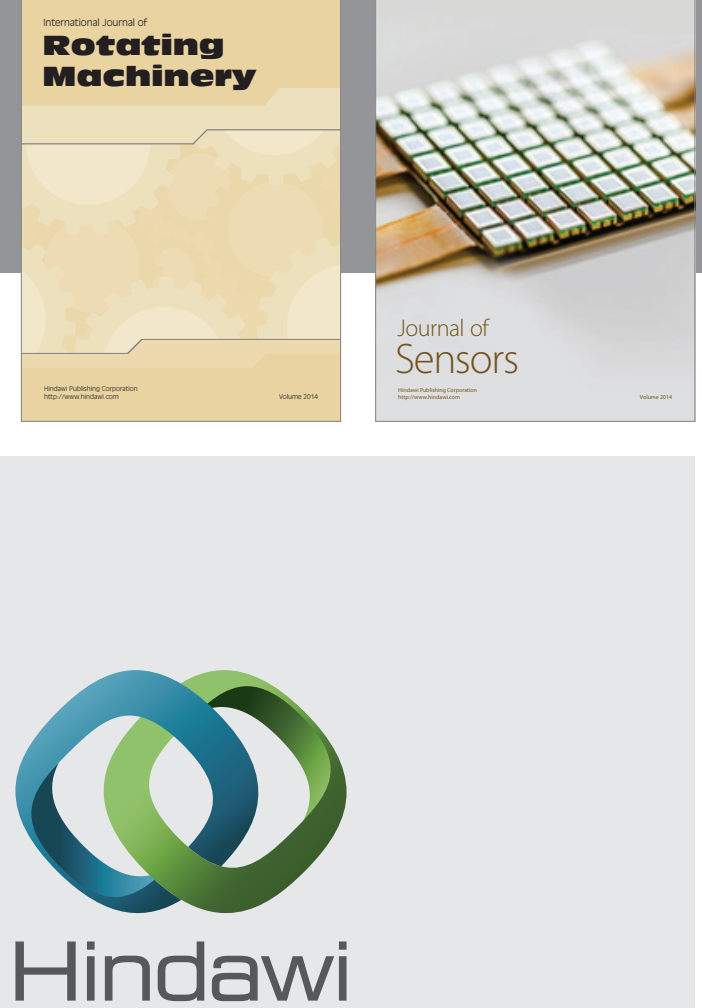

Submit your manuscripts at http://www.hindawi.com
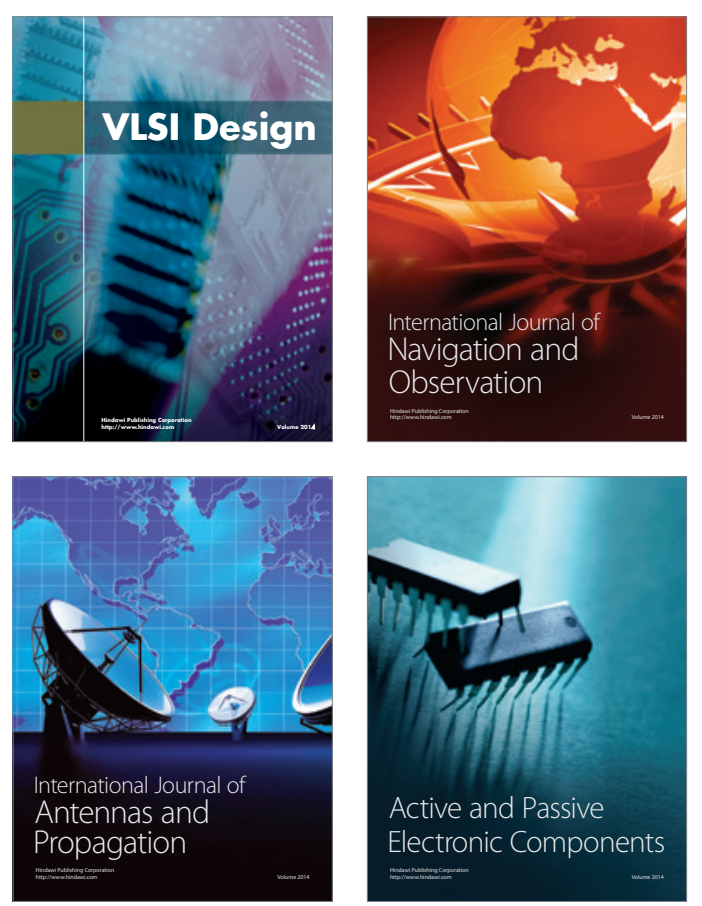
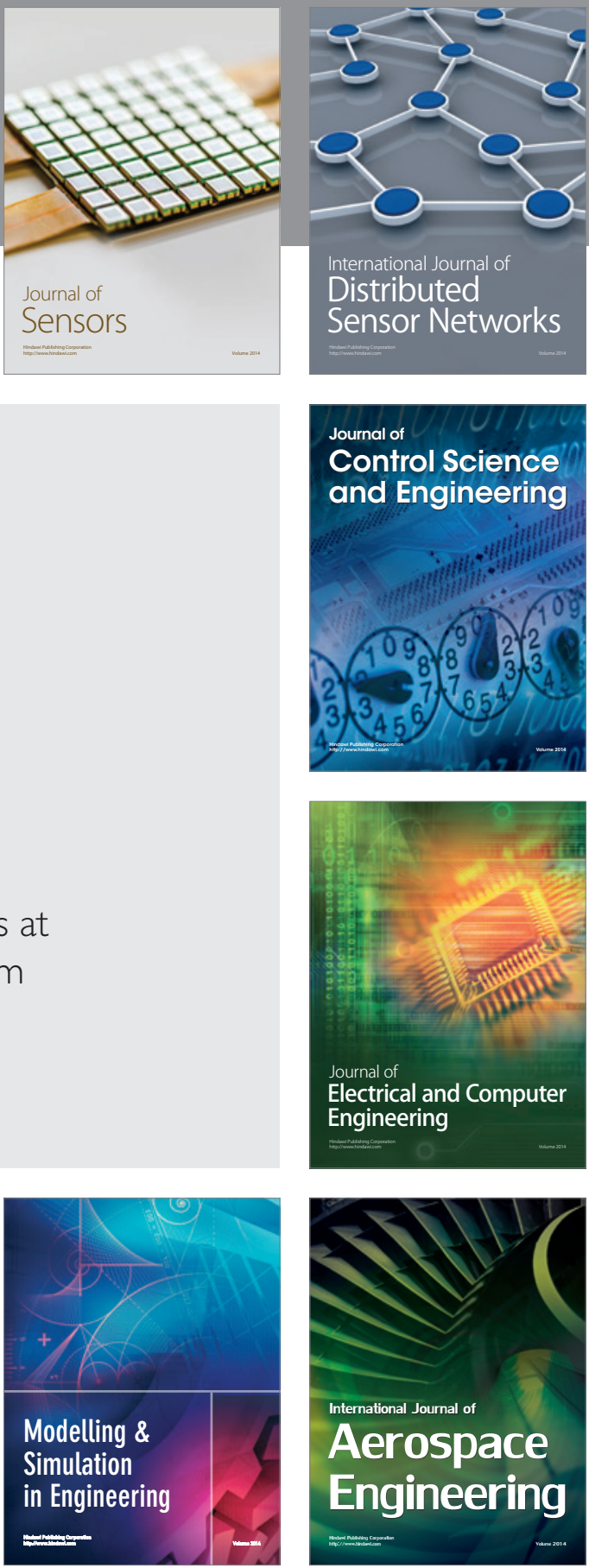

Journal of

Control Science

and Engineering
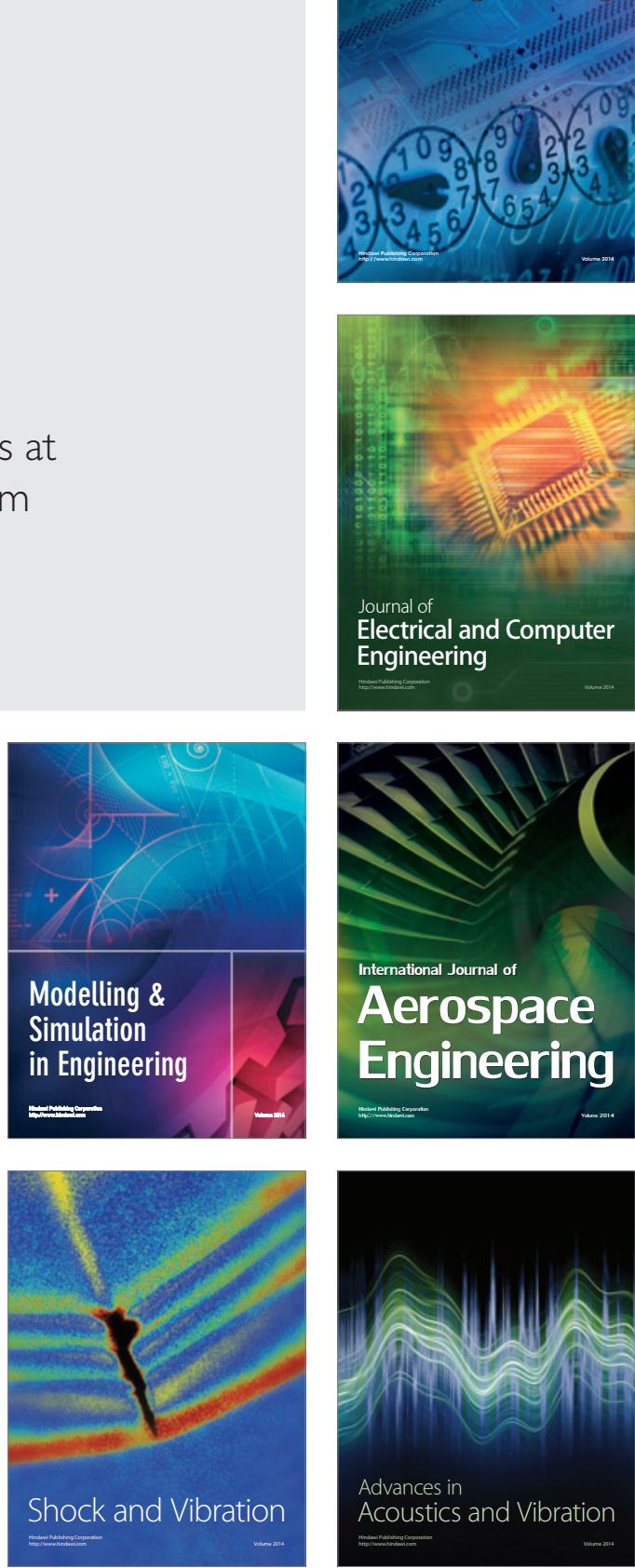